\title{
Hedgehog signalling is essential for maintenance of cancer stem cells in myeloid leukaemia
}

\author{
Chen Zhao ${ }^{1,}{ }^{*}$, Alan Chen ${ }^{1},{ }^{*}$, Catriona H. Jamieson ${ }^{3}$, Mark Fereshteh ${ }^{1}$, Annelie \\ Abrahamsson ${ }^{3}$, Jordan Blum ${ }^{1}$, Hyog Young Kwon ${ }^{1}$, Jynho Kim ${ }^{4}$, John P. Chute ${ }^{2}$, David \\ Rizzieri $^{2}$, Michael Munchhof ${ }^{5}$, Todd VanArsdale ${ }^{6}$, Philip A. Beachy ${ }^{4}$, and Tannishtha Reya ${ }^{1}$ \\ ${ }^{1}$ Department of Pharmacology and Cancer Biology, Duke University Medical Center, Durham, North \\ Carolina 27710, USA. \\ ${ }^{2}$ Department of Medicine, Division of Cellular Therapy, Duke University Medical Center, Durham, \\ North Carolina 27710, USA. \\ ${ }^{3}$ Department of Medicine, Stem Cell Research Program, Moores UCSD Cancer Center La Jolla, \\ California 92093, USA. \\ ${ }^{4}$ Department of Developmental Biology Institute of Stem Cell Biology and Regenerative Medicine, \\ Howard Hughes Medical Institute, Stanford University Medical Center, Stanford, California 94305, \\ USA. \\ ${ }^{5}$ Division of Medicinal Chemistry, Pfizer Laboratories, Groton, Coneecticut 06340, USA. \\ ${ }^{6}$ Division of Oncology, Pfizer Laboratories, La Jolla, California 92121, USA.
}

\section{Abstract}

\begin{abstract}
Although the role of Hedgehog (Hh) signalling in embryonic pattern formation is well established $^{1}$, its functions in adult tissue renewal and maintenance remain unclear, and the relationship of these functions to cancer development has not been determined. Here we show that the lossof Smoothened (Smo), an essential component of the Hh pathway ${ }^{2}$, impairs haematopoietic stem cell renewal and decreases induction of chronic myelogenous leukaemia (CML) by the BCRABL1 oncoprotein ${ }^{3}$. Loss of Smo causes depletion of CML stem cells - the cells that propagate the leukaemia-whereas constitutively active Smo augments CML stem cell number and accelerates disease. As a possible mechanism for Smo action, we show that the cell fate determinant Numb, which depletes CML stem cells, is increased in the absence of Smo activity. Furthermore, pharmacological inhibition of Hh signalling impairs not only the propagation of CML driven by wildtype BCR-ABL1, but also the growth of imatinib-resistant mouse and human CML. These data indicate that Hh pathway activity is required for maintenance of normal and neoplastic stem cells of the haematopoietic system and raise the possibility that the drug resistance and disease recurrence associated with imatinib treatment of $\mathrm{CML}^{4,5}$ might be avoided by targeting this essential stem cell maintenance pathway.
\end{abstract}

(C2009 Macmillan Publishers Limited. All rights reserved

Correspondence and requests for materials should be addressed to T.R. (t.reya@duke.edu)..

*These authors contributed equally to this work.

Author Information Reprints and permissions information is available at www.nature.com/reprints.

Full Methods and any associated references are available in the online version of the paper at www.nature.com/nature.

Supplementary Information is linked to the online version of the paper at www.nature.com/nature.

The authors declare competing financial interests: details accompany the full-text HTML version of the paper at www.nature.com/nature. 
CML arises owing to a translocation between the BCR serine/threonine kinase gene and the ABL1 tyrosine kinase ${ }^{3}$. Imatinib mesylate, which binds to the ABL1 kinase domain and inhibits phosphorylation of substrates, has been used to treat CML, but it is not curative because the cancer stem cells that propagate the leukaemia are resistant to therapy and are not eradicated ${ }^{5-7}$. Furthermore, imatinib resistance due to mutations in the drug-binding site can occur, especially in advanced stage disease, leading to disease relapse and progression ${ }^{4}$. The design of effective new therapies thus critically depends on the identification of signals that are required for CML propagation and in particular signals required for CML cancer stem cell maintenance.

Given the parallels in signalling between development and cancer ${ }^{8,9}$, we examined whether $\mathrm{Hh}$ signalling, an important regulator of development and oncogenesis in many tissues ${ }^{1,10}$, might also have a role in CML. To this end we conditionally deleted Smo-a protein that is essential for Hh signal transduction ${ }^{2}$. Smo is negatively regulated by the Hh receptor Patched (Ptch) in the absence of Hh protein signals. This inhibition is relieved when Ptch is bound by the Hh proteins Shh, Ihh or Dhh. Subsequently, Smo causes activation of Hh pathway targets via the Gli family of transcriptional effectors ${ }^{2}$.

Smo was deleted in the haematopoietic system by crossing mice carrying a loxP-flanked $S m o$ allele ${ }^{11}$ to mice in which Cre is driven by Vav regulatory elements ${ }^{12}$. Smo was efficiently deleted in haematopoietic cells of Vav-Cre;SmoloxP/loxP mice as compared to control $S m o^{\text {loxP/loxP }}$ mice lacking Cre (Fig. 1a, b). Although the frequency of haematopoietic stem cells (HSCs) and differentiated cells was unchanged in the Smo-deficient $\left(\mathrm{Smo}^{-1-}\right)$ mice (Supplementary Fig. 1), these animals had a clear defect in long-term HSC function in primary and secondary transplants (Fig. 1c and Supplementary Fig. 1). Decreased reconstitution was not due to decreased homing or to a preferential loss of any specific lineage (Supplementary Figs 1 and 2), and could be recapitulated with whole bone marrow transplants (Supplementary Fig. 3). Cumulatively, these data indicate that Smo is required for HSC renewal in vivo.

We next investigated the role of $\mathrm{Hh}$ signalling in CML initiation and propagation. CML can be modelled by transplanting BCR-ABL1-transduced haematopoietic progenitors into irradiated mice ${ }^{13}$. Whereas control cells transduced with BCR-ABL1 caused CML in 94\% (16 out of 17) of recipients within 3 months, similarly transduced $\mathrm{Smo}^{-/-}$cells caused CML in only $47 \%$ (8 out of 17) of recipients (Fig. 1d), and these tumours showed an increased latency. The reduced CML incidence was not due to altered homing or engraftment (Supplementary Fig. 4). These data suggest that Hh activity is required for the initiation and propagation of CML. The propagation of several cancers has been shown to depend on cancer stem cells, a critical subset of cancer cells that are capable of transferring the disease to a new host ${ }^{8}$. Because deletion of Smo led to impaired CML growth, we postulated that the loss of Smo might affect CML stem cells. As shown in Fig. 1e, f, the frequency of c-Kit ${ }^{+} \mathrm{Lin}^{-} \mathrm{Sca}-1^{+}(\mathrm{KLS})$ cells (which have previously been shown to be responsible for propagating $\mathrm{CML}^{6}$ ) was significantly reduced in the absence of Smo.

The fact that loss of Smo led to decreased numbers of CML stem cells led us to investigate whether activation of the Hh pathway might lead to an increased frequency of CML stem cells and acceleration of disease. To test this, we used a transgenic mouse expressing an activated form of Smo (SmoM2) fused to yellow fluorescent protein (YFP) ${ }^{14}$; expression of this gene from the Rosa26 locus promoter is blocked by a loxP-flanked stop sequence, and is thus inducible by Cre. When SmoM2-YFP mice were crossed with Vav-Cre mice, progeny expressed YFP in $>70 \%$ of $\mathrm{c}-\mathrm{Kit}^{+} \mathrm{Lin}^{-/ \mathrm{lo}} \mathrm{Sca}-1^{+} \mathrm{Flk} 2^{-}$(KLSF) cells (Fig. 2a). Although the infection of both control and SmoM2 KLSF cells with BCR-ABL1 resulted in CML, the frequency of CML stem cells in animals receiving SmoM2 cells was fourfold greater than in mice receiving control cells (Fig. 2b, c). As expected, SmoM2 was expressed in transgenic but 
not in control CML stem cells (Fig. 2d). Most importantly, the increased CML stem cell frequency led to a significant acceleration of CML progression (Fig. 2e). SmoM2 expression also accelerated CML growth when activated post-embryonically (Supplementary Fig. 5). Thus genetic loss- and gain-of-function experiments indicate that Hh pathway activity controls the frequency and maintenance of CML stem cells, and consequently the incidence and latency of CML formation.

Our previous studies suggested that in normal haematopoietic stem cells, maintenance of the undifferentiated state depends on low levels of the cell fate determinant Numb ${ }^{15}$. We therefore tested whether Numb may act similarly in CML stem cells, and whether the effects of loss of Smo might be due to altered levels of Numb. We found that a greater frequency of Smo ${ }^{-/-}$

CML KLS cells had high levels of Numb as compared to control CML KLS cells (Fig. 3a-c). Additionally, ectopically expressed Numb inhibited in vitro propagation of BCR-ABL1infected haematopoietic cells (Fig. 3d) and of CML stem cells from established leukaemias (Fig. 3e). These data suggest that increased Numb expression may contribute to the loss of CML stem cells in the absence of Smo.

Our genetic data showing that CML stem cells depend on Hh pathway activity raised the possibility that these cells might be targeted by pharmacological blockade of this pathway. We therefore tested the effect of cyclopamine, which inhibits Hh signalling by stabilizing Smo in its inactive form ${ }^{16}$. Exposure of CML stem cells to cyclopamine at a dose determined to minimize off-target effects and toxicity (Supplementary Fig. 6) led to a twofold inhibition of colony growth (Fig. 4a). This could be recapitulated using the Hh-blocking antibody 5E1 (Supplementary Fig. 7), suggesting that Hh pathway activation in CML may be ligand dependent ${ }^{17,18}$. We also delivered cyclopamine to animals transplanted with BCR-ABL1infected HSCs; whereas all of the control animals succumbed to CML within 4 weeks, $60 \%$ of the cyclopamine-treated mice were still alive after 7 weeks (Fig. $4 \mathrm{~b}$ and Supplementary Fig. 8). Furthermore cyclopamine-treated mice had up to a 14-fold reduction in the CML stem cell population (Fig. 4c, d); consistent with this depletion, leukaemias from cyclopamine-treated mice were unable to propagate disease effectively when transplanted (Supplementary Fig. 9). These data suggest that cyclopamine can target the CML stem cell compartment critical for propagation of CML. Notably cyclopamine was most effective when initiated at early stages after CML establishment (Supplementary Fig. 10).

One rationale for identifying new pathways involved in CML is that targeting these pathways may be used to prevent or overcome imatinib resistance ${ }^{19}$. To test whether cyclopamine could impair the growth of imatinib-resistant CML, we infected cells with virus encoding either wildtype BCR-ABL1 or the T315I mutant ${ }^{4,6,20}$ that is resistant to imatinib and other tyrosine kinase inhibitors used at present. Cells infected with mutant BCR-ABL1 were unresponsive to imatinib (Fig. 4e), but their growth was reduced 2.5 -fold by cyclopamine (Fig. 4e). Progression of drug-resistant CML in vivo was also significantly impaired in the presence of cyclopamine (Fig. 4f) as well as in the genetic absence of Smo (Supplementary Fig. 11). These data indicate that Hh pathway activity is required for maintenance of CML stem cells, and raise the possibility that small molecule inhibitors of the pathway could be useful for targeting both normal and imatinib-resistant CML.

Finally we tested whether targeting this pathway could inhibit the growth of human CML. Freshly isolated primary human blast crisis CML samples expressed SMO (Fig. 4g). To determine whether SMO may functionally contribute to human CML growth, we tested the effect of cyclopamine on both a human CML cell line and patient samples in a methylcellulose assay. Cyclopamine decreased colony formation in an imatinib-resistant human CML cell line (Fig. 4h) and in three independent primary patient samples (Fig. 4i and Supplementary Fig. 12), but not in untransformed cord blood cells (Supplementary Fig. 13). Consistent with this, 
inhibition of SMO by the delivery of a new SMO antagonist also impaired the propagation of imatinib-resistant human CML cells in xenografts (A.A., M.M., T.V.A. and C.H.J., manuscript in preparation). Cumulatively, these data suggest that Hh signalling is important for human CML growth and that it may continue to have a role even as the disease progresses into blast crisis phase.

The studies discussed earlier demonstrate a dependence of normal and neoplastic stem cells on Hh signalling. Previously, mammalian Hh proteins have been reported to preserve and increase the short-term repopulating capacity of human $\mathrm{HSCs}^{21}$. Our genetic loss-of-function studies indicate that intact $\mathrm{Hh}$ signalling is needed for HSC renewal. This requirement may be shared with the nervous system, in which progenitor development is dependent on Hh pathway activation $^{22,23}$. Just as $\mathrm{Hh}$ signalling seems to be required for stem cells, its aberrant activation has been associated with the development of several solid cancers ${ }^{17,18,24,25}$. In the haematopoietic system, Hh signalling has been implicated in the growth of B cell lymphoma ${ }^{26}$ and multiple myeloma ${ }^{27}$. Our observations provide critical genetic evidence for a role of Hh signalling in myeloid leukaemia, and together with previous work, suggest that this pathway may have a broader role in haematological malignancies. Our data are also unique in demonstrating that loss of endogenous cancer stem cells impairs in vivo cancer progression, supporting the notion that the leukaemia stem cells identified by transplantation are the relevant drivers of endogenous cancer growth ${ }^{28-30}$. The real impetus for identification of new pathways that drive cancer is the opportunity to inhibit these pathways instead of, or in addition to, conventional therapies. This is especially true for CML, in which imatinib is incapable of eradicating the disease, not only necessitating its continual use but also increasing the likelihood of resistance and relapse. Our findings that the pharmacological inhibition of Hh signalling can impair growth of CML driven by wild-type and imatinib-resistant BCR-ABL1, as well as human CML, raise the possibility that Hh antagonists may be useful as a therapy for normal and drug resistant CML.

\section{METHODS SUMMARY}

For CML assays, HSCs were infected with MSCV-BCR-ABL1-IRES-GFP or MSCV-IRESGFP (control) and transplanted into recipient mice. CML was assessed by flow cytometry and histopathology. Pharmacological inhibition of Hh was initiated 6-8 days after transplantation by delivering $25 \mathrm{mg} \mathrm{kg}^{-1}$ of cyclopamine twice a day by oral gavage. Treatment was continued for five consecutive days, stopped for two days and then continued once a day until the mice were analysed after signs of leukaemia. For human CML studies, peripheral blood CD $34^{+}$cells were sorted and plated in methylcellulose in tomatidine or cyclopamine.

\section{Supplementary Material}

Refer to Web version on PubMed Central for supplementary material.

\section{Acknowledgments}

We wish to thank R. Wechsler-Reya for critical advice, D. Kioussis for Vav-Cre transgenic mice, A. M. Pendergast, A. Means and B. Hogan for review of the manuscript, S. Li for advice on mouse CML experiments, G. Daley, M. Azam and S. Li for the T315I BCR-ABL1 construct, B. Harvat for cell sorting, and J. Harris, B. Zimdahl, N. D'Amato and S. Honeycutt for experimental help. T.R. is a recipient of a CRI Investigator Award, an EMF New Scholar award and a Leukemia and Lymphoma Society Scholar Award, M.F. is supported by the Duke Molecular Cancer Biology Training grant and C.H.J. is funded by the California Institute of Regenerative Medicine and a sponsored research agreement with Pfizer. This work was also supported by National Institutes of Health grants DK63031, DK072234, AI067798 and the Lisa Stafford Memorial Prize to T.R. 


\section{METHODS}

\section{Mice}

The $\mathrm{Smo}^{-/-}$and $\mathrm{Smo}^{+/+}$mice used were in a mixed $129 \mathrm{X} 1 / \mathrm{SvJ}$ and C57BL/6J background. Transplant recipients (C57BL/6 CD45.1) were 8-10 weeks of age. All mice were bred and maintained on acidified, antibiotic water in the animal care facility at Duke University Medical Center. All animal experiments were performed according to protocols approved by the Duke University Institutional Animal Care and Use Committee.

\section{HSC isolation and analysis}

Isolation of HSCs from bone marrow and their transplantation for in vivo analysis of function were performed as described ${ }^{12}$. For analysis of lineage markers, bone marrow cells from control or $\mathrm{Smo}^{-l-}$ mice were incubated with antibodies to murine Ter119 (also known as Ly76), Mac-1 (Itgam), B220 (Ptprc) and CD3 (Cd3e) (eBiosciences), and analysed by FACS. For the reconstitution assays, bone marrow cells from control, $\mathrm{Smo}^{-l-}$ or SmoM2 mice were stained and analysed for the KLSF cells ${ }^{12}$, and 500 control or $\mathrm{Smo}^{-/-}$KLSF cells were injected along with 200,000 competing bone marrow cells into lethally irradiated CD45.1 recipients.

Multilineage repopulation was assessed at 20 weeks. Secondary repopulation assays were carried out by isolating whole bone marrow from primary recipients originally transplanted with control or $\mathrm{Smo}^{-1-} \mathrm{KLSF}$ cells. Recipient mice were killed and analysed for donor chimaerism at 24 weeks.

\section{Generation and analysis of diseased mice}

HSCs from $\mathrm{Smo}^{-1-}$, SmoM2 or control mice were isolated and cultured overnight in X-vivo with $10 \%$ fetal bovine serum (FBS), $100 \mathrm{ng} \mathrm{ml}^{-1}$ stem cell factor (SCF, also known as KITLG) and $20 \mathrm{ng} \mathrm{ml}^{-1}$ thrombopoietin (THPO) in a 96-well U-bottom plate (50,000 per well). Subsequently, cells were infected with MSCV-BCR-ABL1-IRES-GFP or MSCV-IRES-GFP as a control. After $48 \mathrm{~h}$, different cell doses ranging from 15,000-20,000 $\mathrm{Smo}^{-1-}$ or 5,0008,000 limiting SmoM2 cells were transplanted retro-orbitally along with 200,000 whole bone marrow cells into lethally irradiated allelically mismatched recipients. After transplantation, recipient mice were evaluated daily for signs of morbidity, weight loss, failure to groom and splenomegaly. Premorbid animals were killed and relevant tissues were collected and analysed by flow cytometry and histopathology. For flow cytometric analysis of CML stem cells, leukaemic cells were stained for KLS and analysed on FACS-Vantage (BD Biosciences) and Flowjo software (Tree Star, Inc.).

\section{Cyclopamine treatment of diseased mice}

Mice were given $25 \mathrm{mg} \mathrm{kg}^{-1}$ of cyclopamine twice a day by oral gavage beginning 6-8 days after transplantation. Treatment was continued for five consecutive days, stopped for two days, and then continued once a day until the end of the monitoring period. Mice were visually assessed daily for signs of distress. In mice that showed signs of distress treatment was stopped and continued when they showed recovery.

\section{Methylcellulose colony formation assays}

For the colony formation assays CML KLS GFP ${ }^{+}$cells, or KLSF cells infected with wild-type BCR-ABL1 or T315I BCR-ABL1 were sorted into a 24-well plate with complete methylcellulose medium (Stem Cell Technologies) containing cyclopamine (Toronto Research Chemicals) and/or imatinib (Seqouia Research Chemicals). Colonies were counted 8-10 days 
after plating. For the serial replating assay, cells were collected and counted, and 10,000 cells were replated into 12 -well plates.

\section{Immunofluorescence staining}

Primary CML cells collected from bone marrow and spleen were sorted for KLS and GFP cells by FACS. Cells were cytospun, airdried and fixed in $4 \%$ paraformaldehyde. The primary antibody used was goat anti-Numb (Novus Biologicals), and the secondary antibody used was donkey anti-goat-Alexa Fluor 594 (Molecular Probes). DAPI (Molecular Probes) was added as a nuclear counterstain. Slides were viewed on the Axio Imager (Zeiss) at $\times 630$ magnification. Fluorescence intensity analysis was quantified using Metamorph software (Molecular Devices).

\section{Real-time PCR analysis}

RNA was isolated using RNAqueous-Micro (Ambion) and converted to cDNA using Superscript II (Invitrogen). cDNA concentrations were measured with a fluorometer (Turner Designs) using RiboGreen reagent (Molecular Probes). Quantitative rtPCR was performed using an iCycler (BioRad) by mixing equal amounts of cDNAs, iQ SYBR Green Supermix (BioRad) and gene specific primers. Primer sequences are available on request. All real-time data was normalized to actin.

\section{Human CML samples}

All experiments on human CML samples were carried out with approval from the Duke University Institutional Review Board. Patient samples were either obtained from the Duke Adult Bone Marrow Transplant Clinic, or purchased from Open Biosystems. Mononuclear cells were isolated from peripheral blood samples using density-gradient centrifugation. RNA was isolated using RNAqueous-Micro (Ambion) and was converted to cDNA using Superscript II (Invitrogen). PCR with reverse transcription (RT-PCR) was performed (DNA Engine DYAD) with primer sequences specific to human SMO. Primer sequences are available on request. For all colony formation assays, samples were sorted for $\mathrm{hCD} 34^{+}$cells and plated into a 12-well plate (100,000 cells per well) with complete methylcellulose medium (Stem Cell Technologies) in the presence of tomatidine or cyclopamine $(1 \mu \mathrm{M}$, Toronto Research Chemicals). Colony numbers were counted 14-17 days after plating.

\section{References}

1. Ingham PW, Placzek M. Orchestrating ontogenesis: variations on a theme by sonic hedgehog. Nature Rev. Genet 2006;7:841-850. [PubMed: 17047684]

2. Rohatgi R, Scott MP. Patching the gaps in Hedgehog signalling. Nature Cell Biol 2007;9:1005-1009. [PubMed: 17762891]

3. Ren R. Mechanisms of BCR-ABL in the pathogenesis of chronic myelogenous leukaemia. Nature Rev. Cancer 2005;5:172-183. [PubMed: 15719031]

4. Gorre ME, et al. Clinical resistance to STI-571 cancer therapy caused by BCRABL gene mutation or amplification. Science 2001;293:876-880. [PubMed: 11423618]

5. Graham SM, et al. Primitive, quiescent, Philadelphia-positive stem cells from patients with chronic myeloid leukemia are insensitive to STI571 in vitro. Blood 2002;99:319-325. [PubMed: 11756187]

6. $\mathrm{Hu} \mathrm{Y}$, et al. Targeting multiple kinase pathways in leukemic progenitors and stem cells is essential for improved treatment of $\mathrm{Ph}^{+}$leukemia in mice. Proc. Natl Acad. Sci. USA 2006;103:16870-16875. [PubMed: 17077147]

7. Jorgensen HG, Allan EK, Jordanides NE, Mountford JC, Holyoake TL. Nilotinib exerts equipotent antiproliferative effects to imatinib and does not induce apoptosis in CD34 ${ }^{+} \mathrm{CML}$ cells. Blood 2007;109:4016-4019. [PubMed: 17213283] 
8. Reya T, Morrison SJ, Clarke MF, Weissman IL. Stem cells, cancer, and cancer stem cells. Nature 2001;414:105-111. [PubMed: 11689955]

9. Taipale J, Beachy PA. The Hedgehog and Wnt signalling pathways in cancer. Nature 2001;411:349354. [PubMed: 11357142]

10. Evangelista M, Tian H, de Sauvage FJ. The hedgehog signaling pathway in cancer. Clin. Cancer Res 2006;12:5924-5928. [PubMed: 17062662]

11. Long F, Zhang XM, Karp S, Yang Y, McMahon AP. Genetic manipulation of hedgehog signaling in the endochondral skeleton reveals a direct role in the regulation of chondrocyte proliferation. Development 2001;128:5099-5108. [PubMed: 11748145]

12. Zhao C, et al. Loss of b-catenin impairs the renewal of normal and CML stem cells in vivo. Cancer Cell 2007;12:528-541. [PubMed: 18068630]

13. Daley GQ, Van Etten RA, Baltimore D. Induction of chronic myelogenous leukemia in mice by the P210 ${ }^{\text {bcr/abl }}$ gene of the Philadelphia chromosome. Science 1990;247:824-830. [PubMed: 2406902]

14. Mao J, et al. A novel somatic mouse model to survey tumorigenic potential applied to the Hedgehog pathway. Cancer Res 2006;66:10171-10178. [PubMed: 17047082]

15. Wu M, et al. Imaging hematopoietic precursor division in real time. Cell Stem Cell 2007;1:541-554. [PubMed: 18345353]

16. Chen JK, Taipale J, Cooper MK, Beachy PA. Inhibition of Hedgehog signaling by direct binding of cyclopamine to Smoothened. Genes Dev 2002;16:2743-2748. [PubMed: 12414725]

17. Thayer SP, et al. Hedgehog is an early and late mediator of pancreatic cancer tumorigenesis. Nature 2003;425:851-856. [PubMed: 14520413]

18. Watkins DN, et al. Hedgehog signalling within airway epithelial progenitors and in small-cell lung cancer. Nature 2003;422:313-317. [PubMed: 12629553]

19. O'Hare T, Corbin AS, Druker BJ. Targeted CML therapy: controlling drug resistance, seeking cure. Curr. Opin. Genet. Dev 2006;16:92-99. [PubMed: 16343892]

20. Azam M, Latek RR, Daley GQ. Mechanisms of autoinhibition and STI-571/ imatinib resistance revealed by mutagenesis of BCR-ABL. Cell 2003;112:831-843. [PubMed: 12654249]

21. Bhardwaj G, et al. Sonic hedgehog induces the proliferation of primitive human hematopoietic cells via BMP regulation. Nature Immunol 2001;2:172-180. [PubMed: 11175816]

22. Balordi F, Fishell G. Mosaic removal of hedgehog signaling in the adult SVZ reveals that the residual wild-type stem cells have a limited capacity for self-renewal. J. Neurosci 2007;27:14248-14259. [PubMed: 18160632]

23. Han YG, et al. Hedgehog signaling and primary cilia are required for the formation of adult neural stem cells. Nature Neurosci 2008;11:277-284. [PubMed: 18297065]

24. Goodrich LV, Milenkovic L, Higgins KM, Scott MP. Altered neural cell fates and medulloblastoma in mouse patched mutants. Science 1997;277:1109-1113. [PubMed: 9262482]

25. Johnson RL, et al. Human homolog of patched, a candidate gene for the basal cell nevus syndrome. Science 1996;272:1668-1671. [PubMed: 8658145]

26. Dierks C, et al. Essential role of stromally induced hedgehog signaling in B-cell malignancies. Nature Med 2007;13:944-951. [PubMed: 17632527]

27. Peacock CD, et al. Hedgehog signaling maintains a tumor stem cell compartment in multiple myeloma. Proc. Natl Acad. Sci. USA 2007;104:4048-4053. [PubMed: 17360475]

28. Kelly PN, Dakic A, Adams JM, Nutt SL, Strasser A. Tumor growth need not be driven by rare cancer stem cells. Science 2007;317:337. [PubMed: 17641192]

29. Kennedy JA, Barabe F, Poeppl AG, Wang JC, Dick JE. Comment on "Tumor growth need not be driven by rare cancer stem cells". Science 2007;318:1722. doi: 10.1126/science.1149590. [PubMed: 18079385]

30. Adams JM, Kelly PN, Dakic A, Nutt SL, Strasser A. Reply. Science 2007;318:1722. doi: 10.1126/ science. 1149672 . 

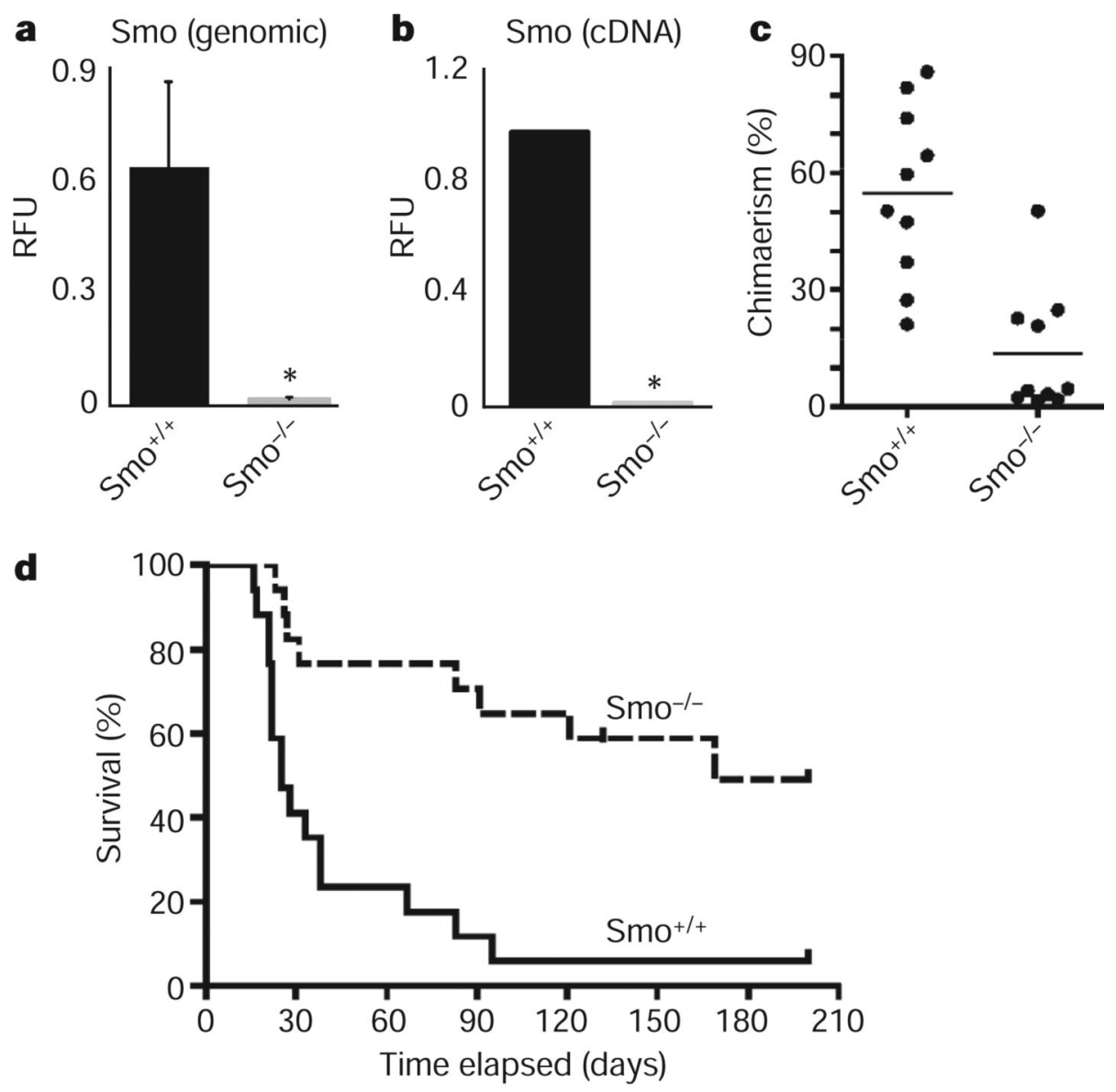

e
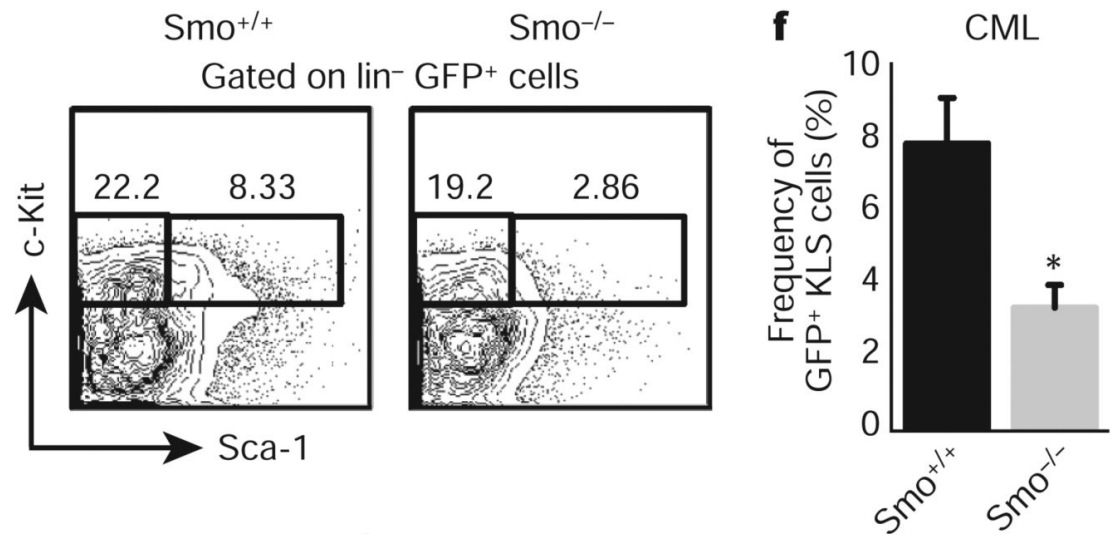

Figure 1. Conditional deletion of Smo impairs the development of BCR-ABL1-induced CML and depletes CML stem cells

a, Real-time PCR (rtPCR) using primers specific for the loxP sites was carried out on $\mathrm{Smo}^{-/-}$or $\mathrm{Smo}^{+/+}$bone marrow genomic DNA $(n=3, * P=0.03)$. RFU, relative fluorescent units. b, rtPCR for $S m o$ using complementary DNA from $S m o^{-/-}$or $S m o^{+/+}$KLSF cells $(n=$ 7, $* P<0.00001)$. c, KLSF cells from $\mathrm{Smo}^{-/-}$or $\mathrm{Smo}^{+/+}$mice were transplanted together with competing bone marrow cells into congenic recipients and donor-derived chimaerism was monitored. The graph shows the average donor-derived chimaerism after long-term reconstitution ( $n=2$ with 20 mice, $P=0.0002)$. d, Survival curve of mice transplanted with BCR-ABL1-infected $\mathrm{Smo}^{-/-}$or $\mathrm{Smo}^{+/+} \mathrm{KLSF}$ cells $(n=3$ with 34 mice, $P=0.0002)$. e, 
Representative example of CML stem cells $\left(\mathrm{GFP}^{+} \mathrm{Lin}^{-} \mathrm{c}-\mathrm{Kit}^{+} \mathrm{Sca}^{+}\right)$in the bone marrow of mice transplanted with BCR-ABL1-infected $\mathrm{Smo}^{-/-}$or $\mathrm{Smo}^{+/+} \mathrm{KLS}$ cells. f, Graph of the average percentage of CML stem cells in mice receiving BCR-ABL1-infected $\mathrm{Smo}^{+/+}(8$ mice $)$ or $\mathrm{Smo}^{-l-} \mathrm{KLS}$ cells (9 mice). Frequency shows $\mathrm{c}-\mathrm{Kit}^{+} \mathrm{Sca}^{+}$cells as as a percentage of the $\mathrm{GFP}^{+} \mathrm{Lin}^{-}$population. $* P=0.006$. Error bars in all bar graphs are s.e.m. 

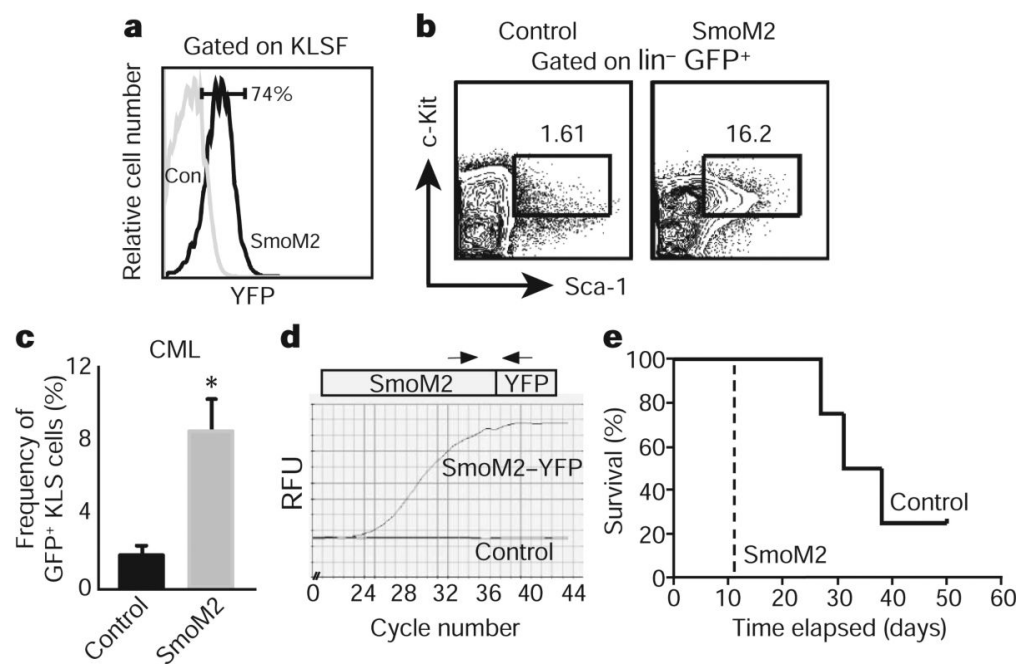

Figure 2. The presence of constitutively active Smo increases the frequency of CML stem cells and accelerates disease

a, Analysis of YFP fluorescence, which reflects SmoM2 expression in control (con) and SmoM2 KLSF cells $(n=4)$. b, Analysis of CML stem cells $\left(\mathrm{GFP}^{+} \mathrm{KLS}\right)$ in mice transplanted with BCR-ABL1-infected control (left) and constitutively activated Smo (SmoM2, right)

KLSF cells. c, The average percentage of CML stem cells in mice receiving BCR-ABL1transduced control $(n=4)$ and SmoM2 KLSF cells $(n=12)$. Error bars are s.e.m., $* P=0.048$. d, rtPCR analysis of SmoM2 expression in CML stem cells. e, Survival curve of mice receiving BCR-ABL1 transduced control (solid line) or SmoM2 KLSF cells (dashed line; $n=2,16$ mice, $P=0.0082)$. 

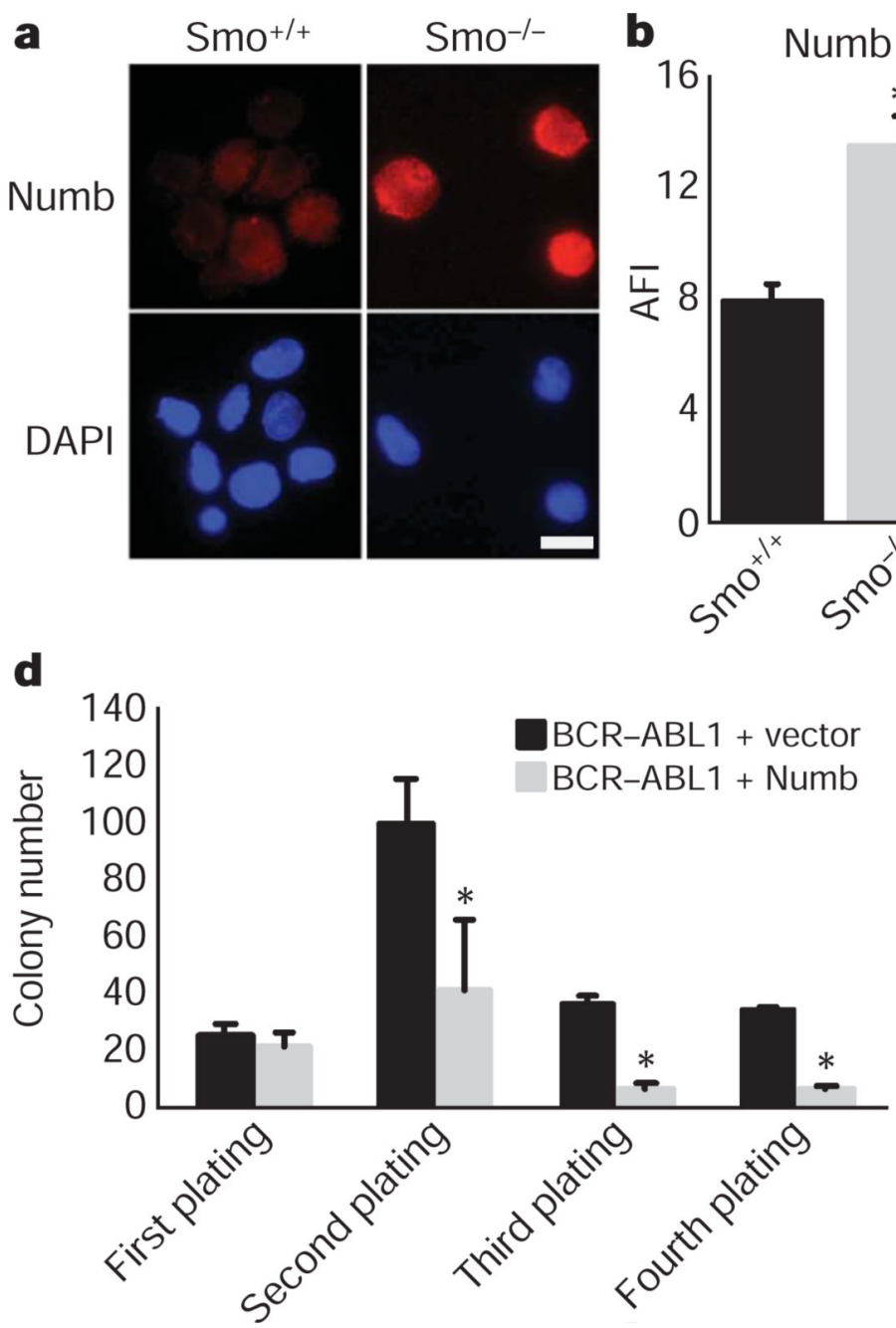
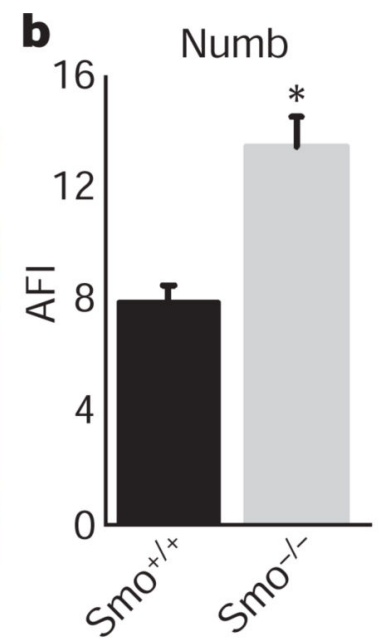

e
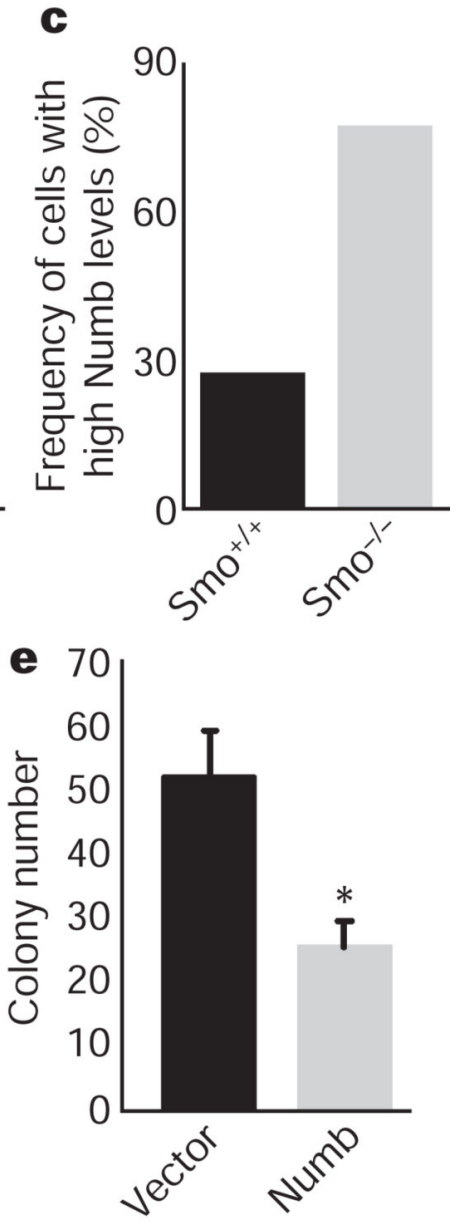

Figure 3. Loss of Smo increases frequency of cells with high levels of Numb and contributes to decreased CML growth

a, CML stem cells from $\mathrm{Smo}^{+/+}$and $\mathrm{Smo}^{-/-}$leukaemias were stained with Numb (red, top panel) and 4,6-diamidino-2-phenylindole (DAPI; blue, bottom panel). Scale bar, $10 \mu \mathrm{M}$. b, The average fluorescence intensity (AFI) was determined by dividing the overall mean fluorescence intensity by the area of the cell $(P=0.002)$. c, The frequency of cells with high expression levels of Numb was calculated by designating cells above a mean fluorescence intensity value of 1,000 as high expressors ( $n=3$, using either CML KLS or CML c-Kit ${ }^{+}$cells). d, KLSF cells were infected with MSCV-BCR-ABL1-IRES-GFP and either vector MSCVIRES-YFP or MSCV-Numb-IRES-YFP. GFP and YFP double-positive cells were plated in methylcellulose, colonies were counted and cells were serially replated $(n=2, * P<0.006)$. e, CML KLS cells were infected with viruses expressing control vector or Numb IRES-YFP. Infected cells were sorted and plated in methylcellulose $(n=2, P=0.03)$. Error bars in $\mathbf{b}-\mathbf{e}$ are s.e.m. 


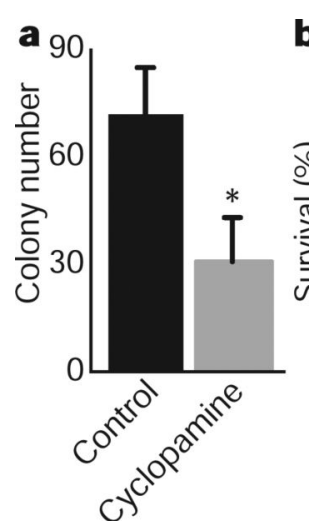

e

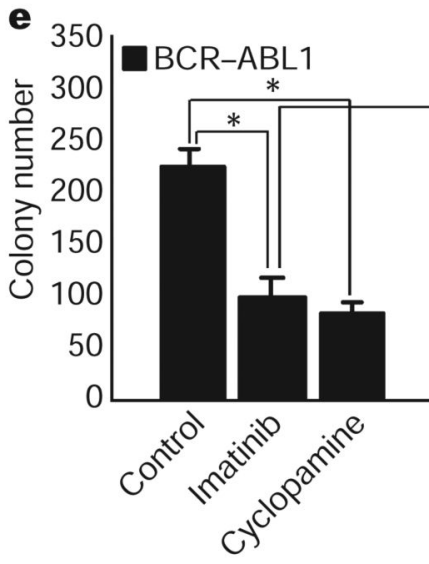

$\mathbf{g}$

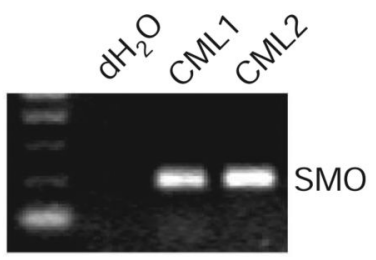

\section{b}

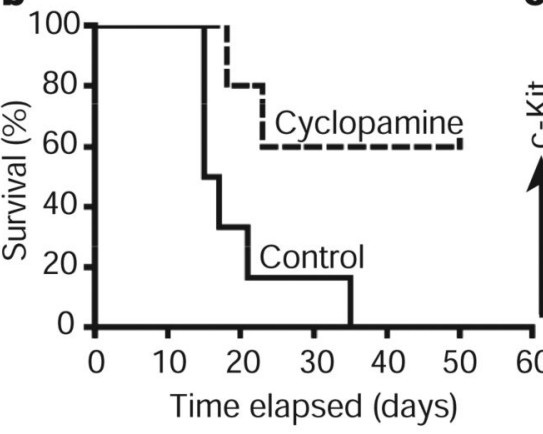

c

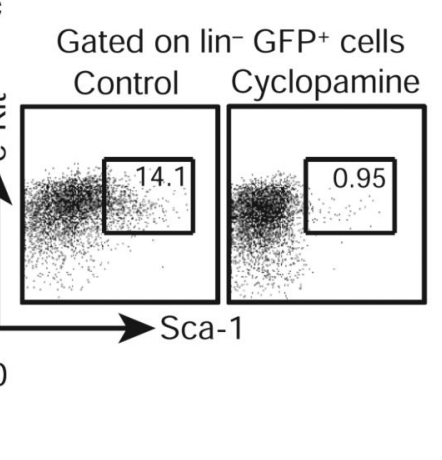

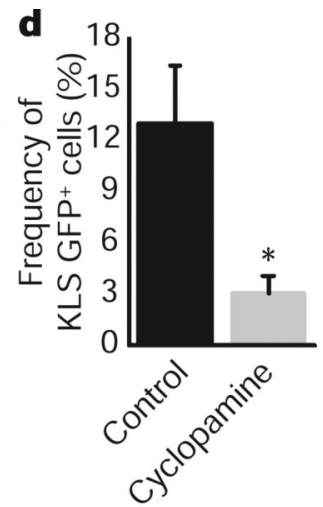

Figure 4. Pharmacological inhibition of Smo impairs CML development and propagation a, CML stem cells were plated in methylcellulose in the presence of $1 \mu \mathrm{M}$ tomatidine (control) or cyclopamine $(n=2, * P=0.005)$. $\mathbf{b}$, Survival curve of mice transplanted with BCR-ABL1infected KLS cells in the presence of vehicle or cyclopamine ( $n=3$ with 32 mice, $P=0.02$ ). c, Representative example of CML stem-cell frequency in vehicle- or cyclopamine-treated CML. d, Average CML stem cell frequency in vehicle-or cyclopamine-treated mice $(n=4$, $* P=0.03)$. e, KLSF cells were infected with wild-type BCR-ABL1 or the imatinib-resistant T315I mutant BCR-ABL1, and plated in methylcellulose with imatinib $(2 \mu \mathrm{M})$ or cyclopamine $(1 \mu \mathrm{M})\left(n=2,{ }^{*} P<0.004\right)$. f, Survival curve of mice receiving T3151 BCR-ABL1-infected KLSF cells and either vehicle or cyclopamine, 7 days after transplantation $(n=2$ with 16 mice, $P=0.021)$. g, $S M O$ PCR on primary uncultured human CML samples. h, Imatinib-resistant K562 blast crisis CML cells were plated in methycellulose in the presence of $1 \mu \mathrm{M}$ tomatidine or cyclopamine $(n=2, * P<0.01)$. i, CD $34^{+}$cells were sorted from primary uncultured human 
blast crisis CML samples and plated in methylcellulose in the presence of $1 \mu \mathrm{M}$ tomatidine or cyclopamine $(n=3, * P<0.05)$. Error bars in all bar graphs are s.e.m. 